\title{
Retinoblastoma: report of a case with minimal retinal involvement but massive anterior segment spread
}

\author{
ALEC GARNER,' JACK J KANSKI, ${ }^{2}$ AND FIONA KINNEAR ${ }^{2}$ \\ From the 'Department of Pathology, Institute of Ophthalmology, University of London, and the ${ }^{2}$ King Edward \\ VII Hospital, Windsor
}

SUMmaRY The case is described of a unilateral retinoblastoma in a 7-year-old girl which was initially misdiagnosed because of massive anterior segment involvement in the apparent absence of a retinal lesion. Only on histopathological examination of the enucleated eye was the presence of retinal tumour established, this being confined to two minute foci at the extreme periphery.

Invasion of the anterior segment by retinoblastoma is a well recognised if not very common complication, and in that it may simulate ocular inflammation it is a significant source of misdiagnosis. ${ }^{2}$ Seeding from an endophytic tumour is the usual cause of such spread, but appreciation of the true nature of the disease process can be difficult if associated cataract formation or vitreous exudates interfere with examination of the posterior segment. Nevertheless, given a proper index of suspicion, recourse to alternative methods of investigation such as ultrasonography and CT scanning should lead to the correct diagnosis. More difficult is the much rarer diffuse infiltrating growth pattern,,$^{34}$ since the retina may appear clinically normal despite adequate visualisation.

In this report we describe a child with massive anterior segment invasion in which the retinal involvement was confined to two minute foci at the extreme periphery. The paucity of retinal involvement is unprecedented in our experience of over 1000 retinoblastoma cases and we know of only one other case in the literature to invite comparison. ${ }^{5}$

\section{Case history}

A 7-year-old girl presented in March 1985 complaining of redness and blurring of vision of her right eye for about four weeks. On examination visual acuity was reduced to $6 / 60$. Slit-lamp biomicroscopy showed a severe anterior uveitis with large iris nodules (Fig. 1) and cells and opacities in the anterior vitreous. Investigations, all of which were negative, included a

Correspondence to Professor A Garner, Institute of Ophthalmology, 17/25 Cayton Street, London EC1V 9AT. full blood count, plasma viscosity, toxoplasma dye test, and conjunctival biopsy for sarcoidosis. A presumptive diagnosis of granulomatous uveitis was made, and treatment was begun with intensive topical corticosteroids and systemic prednisolone $5 \mathrm{mg}$ three times a day. Subsequently the intraocular pressure became raised to $44 \mathrm{mmHg}$, and because it failed to respond to antiglaucoma therapy with timoptol $0.25 \%$ twice daily, propine $0.1 \%$ twice daily, and acetazolamide $125 \mathrm{mg}$ four times a day a cyclocryotherapy operation was performed at the end of May 1985.

The intraocular inflammation remained extremely active despite an anterior sub-Tenon's capsule injection of methylprednisolone (Depomedrone). In early June 1985 a blood stained hypopyon developed (Fig. 2 ) and the lens became cataractous. At the end of June 1985 it was suspected that the eye might be

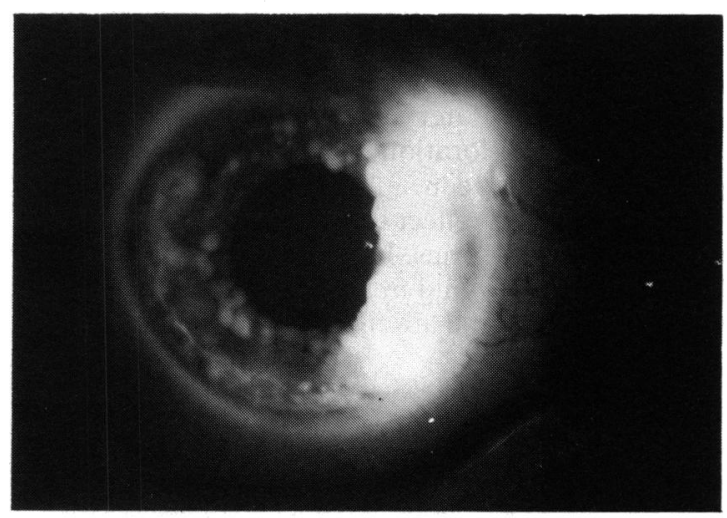

Fig. 1 Nodular infiltration of the iris by retinoblastoma. 


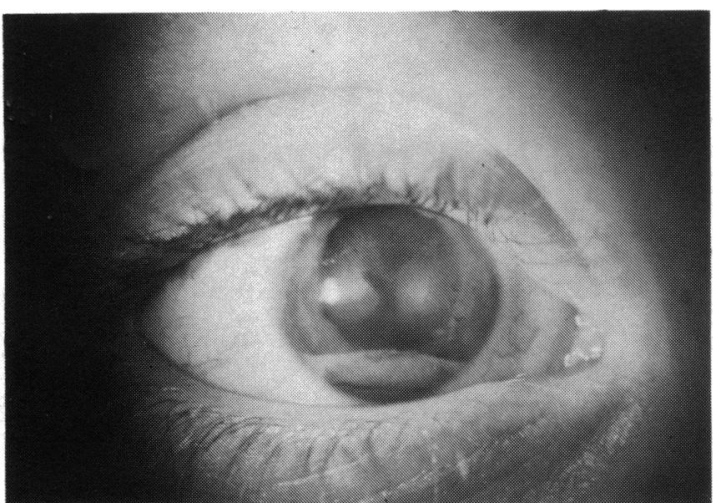

Fig. 2 Blood stained pseudohypopyon in conjunction with retinoblastoma and secondary cataract.

harbouring a malignant tumour, and diagnostic aspirates were taken from the aqueous humour and anterior vitreous. At the same time the iris was biopsied and the lens excised with a vitreous cutter through a pars plana incision. After the lens was removed a mass of white friable tissue was found in the area of the inferior pars plana. Following a preliminary histological report which suggested the presence of a malignant tumour an enucleation was performed two weeks later.

In February 1986 the prosthesis became tilted owing to an orbital swelling which quickly developed into an expanding orbital mass. In mid March 1986 an excision biopsy of the mass was performed and histology showed recurrence of the tumour. The patient was treated by orbital radiotherapy and adjuvant chemotherapy. Staging investigations prior to the institution of chemotherapy showed no

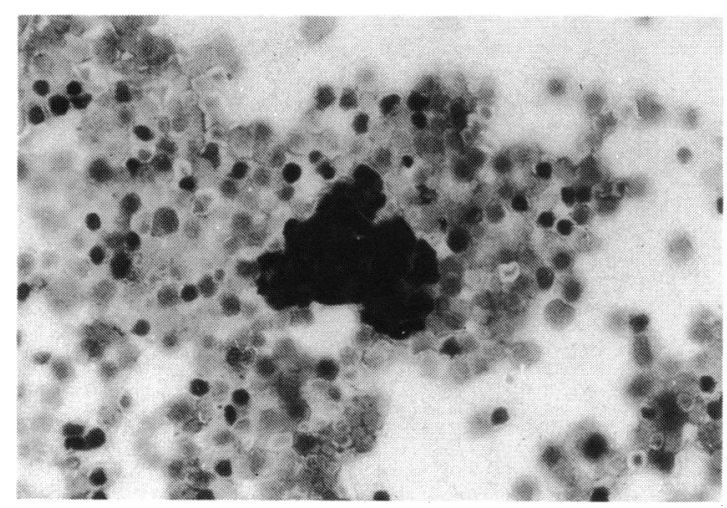

Fig. 3 Aqueous tap showing cells with small, darkly stained nuclei and scanty cytoplasm. Some cells are separate, others are in clumps and many are degenerate. Haematoxylin and eosin. evidence of gross metastatic disease, but monoclonal antibodies on the bone marrow aspirate did show a few cells positive for neuroectodermal markers suggesting possible infiltration of her bone marrow with retinoblastoma cells which were not identifiable on standard light microscopy. Following completion of the initial course of chemotherapy the marrow aspirate was negative for cells of neuroectodermal origin on monoclonal antibody studies. Since then the patient has received two further courses of chemotherapy and is due to have more. In June 1986 she was alive and well, with no sign of further recurrence.

\section{PATHOLOGY}

Initial biopsies. A piece of iris removed at the time of lensectomy showed the stroma to be replaced almost completely by cells that were mostly round with quite strongly basophilic nuclei and hardly any cytoplasm. Some nuclear pyknosis and karyorrhexis were also seen. Samples from the aqueous (Fig. 3) and vitreous humours contained similiar cells, lying both singly and in clumps, and some haemorrhage. Immunohistochemical staining by a peroxidaseantiperoxidase method gave negative responses for cell-bound immunoglobulin using a polyvalent antiserum, for S-100 protein, and for $\gamma$-neuron-specific enolase. Despite the lack of confirmatory evidence from the marker studies of a neuronal derivation a tentative diagnosis of retinoblastoma was made.

Enucleation specimen. There was evidence of a recent superior iridectomy, and the residual iris had an uneven, nodular, and greyish white appearance. Opening the globe in a vertical plane and removing the nasal calotte revealed soft, creamy nodules covering much of the posterior surface of the iris and similar material overlying the ciliary body. A little haemorrhage was observed on the posterior retinal surface and in the anterior vitreous, but otherwise the retina showed no further abnormality on macroscopical examination.

Light microscopy showed that most of the iris stroma and much of the ciliary body was infiltrated and replaced by round cells comparable to those seen in the earlier biopsy (Fig. 4). In places, particularly within the ciliary body, there was a tendency for the cells to form cords or ribbons, but true FlexnerWintersteiner rosettes were not seen (Fig. 5). There were numerous islands of similar cells within the posterior chamber and in the anterior chamber, where they also lined the back of the cornea and occluded the drainage angle. The cells at the centre of some of the larger clumps were necrotic. Sections passing through the site of surgical intervention showed tumour cells in proximity to the scleral incision, but there was no sign of extraocular tumour. 
Fig. 4 Section of the enucleated eye showing massive infiltration of the iris by small, darkly stained tumour cells. Free lying tumour cells in the aqueous, some haemorrhage and a remnant of lens are also seen. Haematoxylin and eosin.

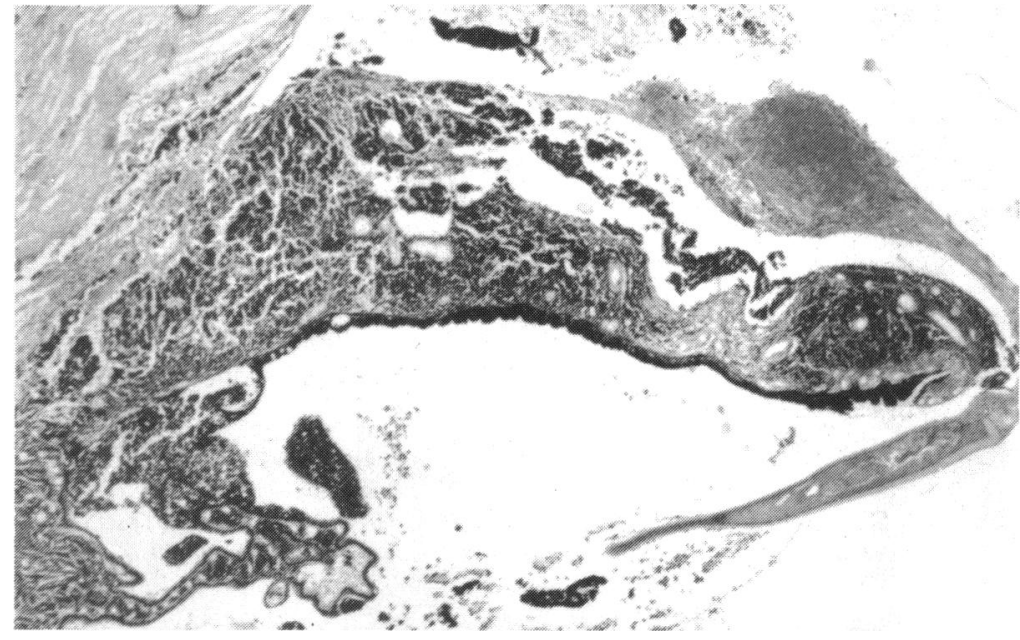

The lens capsule with some residual lens material was adherent to the pupillary margin of the iris, and there was blood in the anterior vitreous. Some atrophy of the corneal epithelium, possibly reflecting the effects of raised intraocular pressure, was also seen. Initial sections of the retina revealed no abnormality (Fig. 6) and it was only after examining multiple levels that two small, discrete foci of cellular proliferation comparable to that seen in the anterior segment were identified between the 11 and 12 o'clock meridians and the 6 and 7 o'clock meridians respectively at the extreme periphery (Figs. 7, 8). Neither focus was more than $1.5 \mathrm{~mm}$ in diameter, and there was minimal thickening of the affected retina. The inferior tumour proliferation was separated from the pars plana ciliaris by about $1.0 \mathrm{~mm}$ of atrophic, acellular retina. Origin of the neoplastic process from the bipolar cell layer appeared possible. Away from the tumour the retina appeared to be normal, as did the optic nerve and choroid.

Immunohistochemistry on paraffin embedded tissue gave a variable but, in places, unmistakeable staining response for $\gamma$-neuron-specific enolase. Reactions for S-100 protein and glial fibrillary acidic protein were negative.

Examination of formalin fixed, free lying cells recovered from the posterior chamber by transmission electron microscopy revealed occasional cilialike structures with a ring of nine doublet microtubular structures but no central component $(9+0)$

Fig. 5 Tumour cells in the ciliary body are forming cords, while those on its surface are randomly arranged with little evidence of rosette formation. Haematoxylin and eosin.

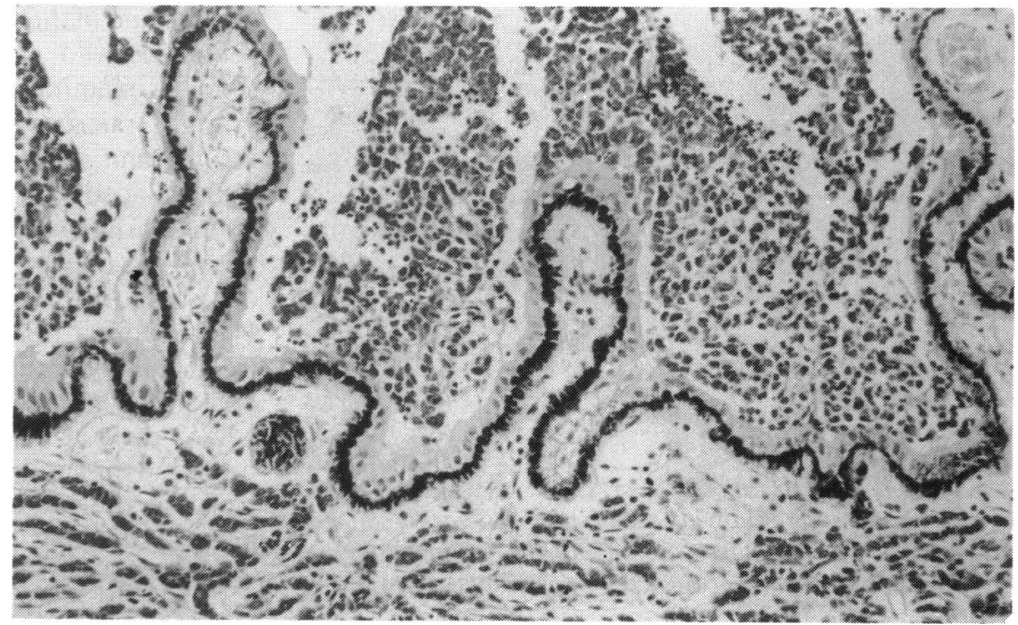




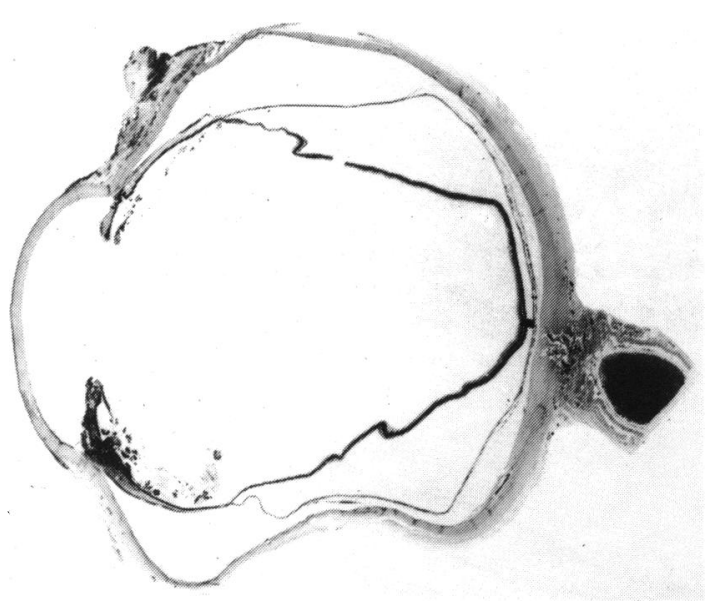

Fig. 6 Vertical section of the enucleated eye shows tumourous infiltration of the iris and ciliary body, particularly inferiorly, but the retina shows no abnormality apart from artefactual detachment. Tumour cells are seen in the posterior chamber and anterior vitreous. Haematoxylin and eosin.

(Fig. 9) and numerous intercellular junctions of intermediate type.

\section{Discussion}

The morphology of the cells at the initial biopsy, while consistent with retinoblastoma, was far from conclusive and warranted the consideration of alternative diagnosis. Given that the clinical diagnosis of uveitis made when the patient first presented was not substantiated by an appropriate response to antiinflammatory drug therapy or the histological character of the cellular infiltrate, the principal alternative was acute lymphocytic or monocytic leukaemia. This would have needed to be in an aleukaemic phase, since examination of the peripheral blood revealed no abnormality, but the absence of other symptoms and signs made leukaemic infiltration an unlikely diagnosis. Moreover, there was no evidence of cell bound immunoglobulin as might have been expected in the case of lymphocytic neoplasia. Nevertheless, the diagnosis of retinoblastoma was to some extent one of exclusion, and the finding of an apparently normal retina when the enucleated eye was opened in the laboratory provoked temporary misgivings, at least, on the part of the pathologist responsible for the biopsy evaluation. The subsequent demonstration of cilialike structures with the $9+0$ configuration characteristic of retinal photoreceptor cells and of many retinoblastomas $^{67}$ served, however, to support the diagnosis, and the finding of intermediate junctions between the cells, while not conclusive evidence of retinoblastoma, excluded a leukaemic process.

The demonstration of neuron-specific enolase in some cells was also indicative of a neuronal tumour. In a study by Terenghi et al. ${ }^{8}$ all but one of 30 retinoblastomas stained for neuron-specific enolase, irrespective of the degree of differentiation, but other reports ${ }^{90}$ suggest that this enzyme is usually confined to the more primitive tumours such as pertained in the present case. The demonstration of S-100 protein is a less reliable marker of retinoblastoma, for, while Terenghi et al. ${ }^{8}$ found it to be present in all but five of the cases they examined, others have provided uniformly negative reports. ${ }^{9}$

Immunohistochemical staining for glial fibrillary acidic protein was negative in the present case, and,

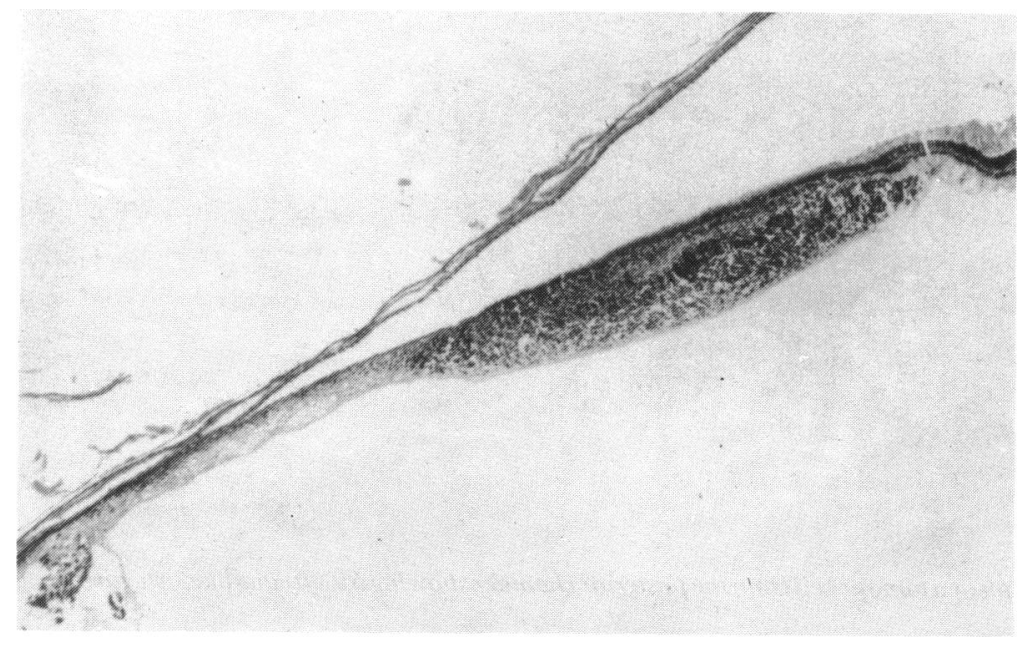

Fig. 7 The peripheral retina in the superior hemisphere shows a little focal increase in thickness due to the proliferation of retinoblastoma cells. Haematoxylin and eosin. 
Fig. 8 A second small focus of tumour is seen at the inferior retinal periphery. Haematoxylin and eosin.

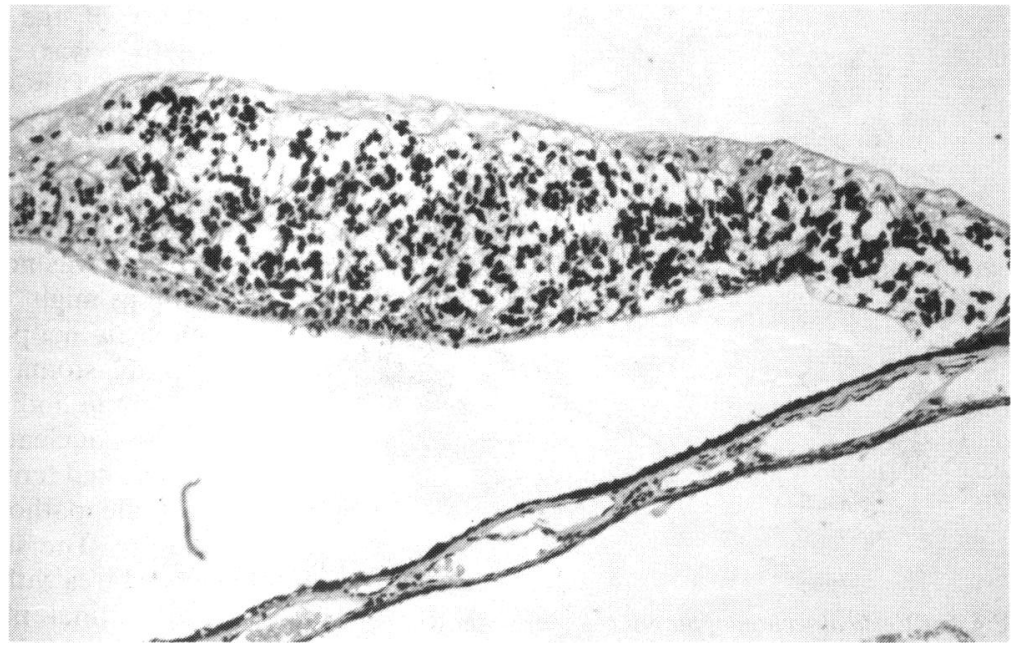

growth patterns of retinoblastoma the case under consideration compares most closely with the diffuse infiltrating type. ${ }^{3413}$ Even so, two isolated foci measuring $1.5 \mathrm{~mm}$ at most, are only partially described by this designation, and the present case must

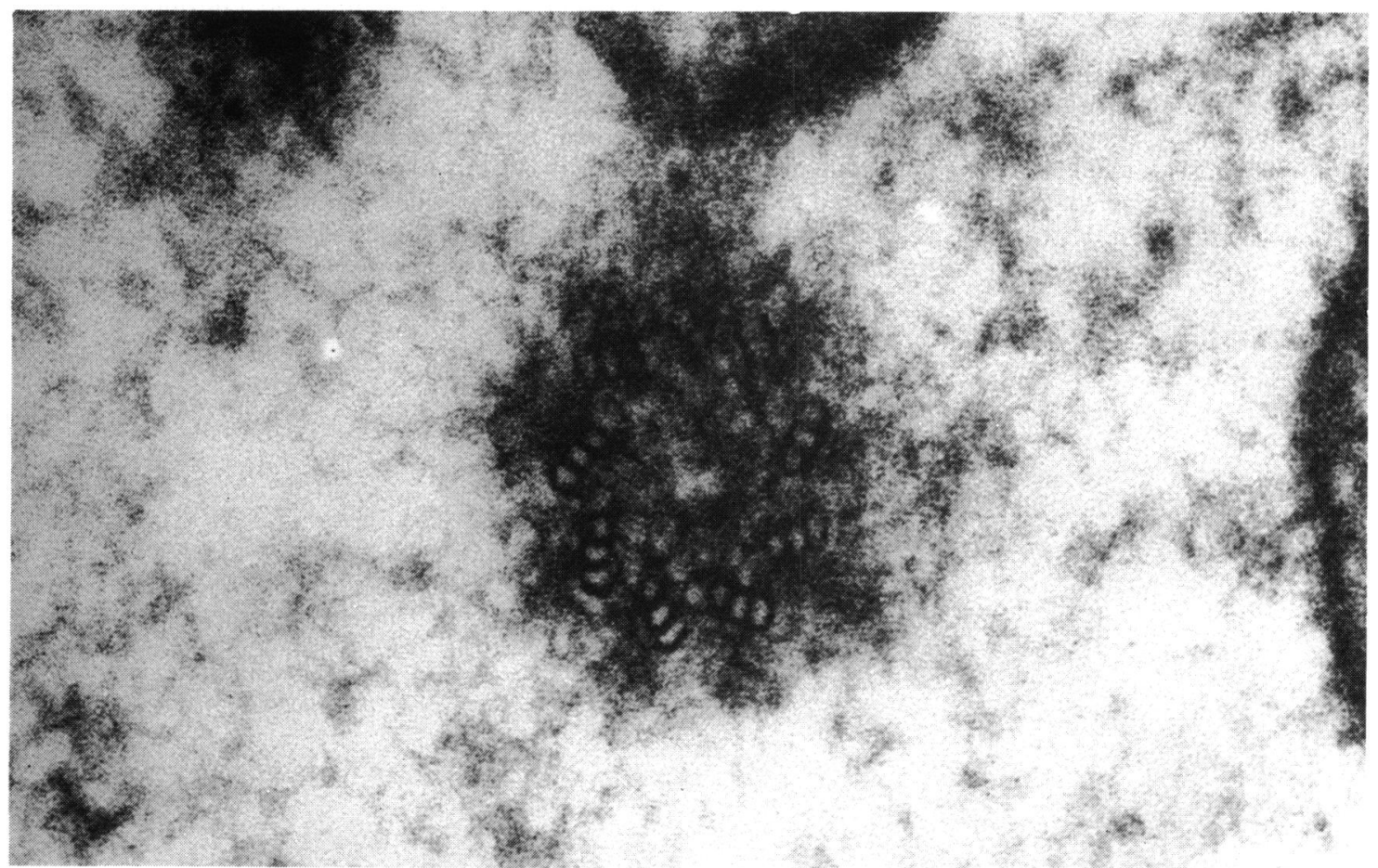

Fig. 9 Transmission electron micrograph of a tumour cell from the posterior chamber showing a $9+0$ cilia-like structure. Uranyl acetatellead citrate, $\times 200000$. 
be regarded as an extreme rarity. The closest analogy in the literature appears to be the case presented by Reeser to the Verhoeff Society in $1975^{5}$ and depicted by Zimmerman, ${ }^{2}$ but examination of the histological section indicates a slightly more extensive intraretinal lesion than pertains in our case. They are, nevertheless, both likely to represent one end of a spectrum of the extent to which the retina can manifest the diffuse infiltrating form of retinoblastoma before presenting with predominantly anterior segment changes. It is noteworthy, although the relevance is obscure, that the age of the patient being reported was 7 years at initial presentation, that Reeser's case was 8 years old, and that seven of the 10 cases of diffuse infiltrating retinoblastoma described by Morgan ${ }^{3}$ ranged between $43 / 4$ and 10 years in age. Morgan ${ }^{3}$ commented that retinoblastomas of the diffuse infiltrating type have a better prognosis than other types, but any prediction in the present case is clouded by recurrence of the tumour in the orbit eight months after enucleation.

We are grateful to Mr J D Griffiths, FRCS, who was responsible for the initial referral and for the enucleation, for permission to report this case, and to Dr A C E McCartney for electron microscopical data.

\section{References}

1 Stafford WR, Yanoff M, Parnell BL. Retinoblastoma misdiagnosed as primary ocular inflammations. Arch Ophthalmol 1969; 82: 771-3.
2 Zimmerman LE. Retinoblastoma and retinocytoma. In: Spencer W, ed. Ophthalmic pathology: an atlas and textbook. 3rd ed. Philadelphia: Saunders, 1985: 1292-351.

3 Morgan G. Diffuse infiltrating retinoblastoma. Br J Ophthalmol 1971; 55: 600-6.

4 Nicholson DH, Norton EW. Diffuse infiltrating retinoblastoma. Trans Am Ophthalmol Soc 1980; 78: 265-89.

5 Reeser F. Iris spread from a peripheral retinoblastoma. Presented at the Verhoeff Society Meeting, Washington, DC, 1975.

6 Popoff N, Ellsworth RM. The fine structure of retinoblastoma: in vivo and in vitro observations. Lab Invest 1971; 25: 389-402.

7 Albert DM, Lahav M, Lesser R, Craft J. Recent observations regarding retinoblastoma $I$. Ultrastructure, tissue culture growth, incidence, and animal models. Trans Ophthalmol Soc UK 1974; 94: 909-28.

8 Terenghi G, Polak JM, Ballesta J, et al. Immunocytochemistry of neuronal and glial markers in retinoblastoma. Virchows Arch (A) 1984; 404: 61-73.

9 Molnar ML, Stefansson K, Marton LS, Tripathi RS, Molnar GK. Immunohistochemistry of retinoblastomas in humans. Am J Ophthalmol 1984; 97: 301-7.

10 Kyritsis AP, Tsokos M, Triche TJ, Chader GJ. Retinoblastoma: origin from a primitive neuroectodermal cell. Nature 1984; 307: 471-3.

11 Jiang Q, Lim R, Blodi FC. Dual properties of cultured retinoblastoma cells: immunohistochemical characterisation of neuronal and glial markers. Exp Eye Res 1984; 39: 207-15.

12 Lane JC, Klintworth GK. A study of astrocytes in retinoblastomas using the immunoperoxidase technique and antibodies to glial fibrillary acidic protein. Am J Ophthalmol 1983; 95: 197-207.

13 Schofield PB. Diffuse infiltrating retinoblastoma. $\mathrm{Br} J$ Ophthalmol 1960; 44: 35-41.

Accepted for publication 31 October 1986. 